\title{
SURGICAL MANAGEMENT OF MESIODENS BASED ON CHARACTERISTICS AND COMPLICATIONS OF THE CONDITION: A SYSTEMATIC REVIEW
}

\author{
Bayu Rahadian, Vera Julia, Lilies D. Sulistyani \\ Department of Oral and Maxillofacial Surgery, Faculty of Dentistry, Universitas Indonesia, Indonesia
}

\begin{abstract}
Disturbances in dental development may result in anomalies, such as supernumerary teeth in the upper or lower jaw. Mesiodens is the most prevalent supernumerary tooth that is often found in the midline of the maxilla and can cause problems, such as malpositioned permanent teeth, diastema formation, cyst formation, and delayed eruption of maxillary anterior incisors. Surgical treatment might be an option for the management of this condition. This study aims to review the characteristics and complications, which need to be considered for the surgical management of mesiodens. A literature search for articles written in English and published within the last 10 years was conducted using the electronic database, PubMed. The keywords verified by Medical Subject Headings included mesiodens, characteristics, complications, and management. A total of 60 articles were retrieved; however, after exclusion, only 45 articles were relevant for evaluation. Subsequently, only 30 articles with full-text versions available online were included in the study. Based on this review, the majority of studies factored in the complications of mesiodens during treatment planning. Very few studies covered the relationship between the characteristics and complications of this condition and its management.
\end{abstract}

KEY WORDs: surgical management, mesiodens, supernumerary teeth, tooth abnormalities, radiographic study.

J Stoma 2020; 73, 5: 261-269

DOI: https://doi.org/10.5114/jos.2020.100583

\section{INTRODUCTION}

Mesiodens is an abnormality in the number of teeth in the upper or lower jaw, which results from disturbances in dental development $[1,2]$. Although the etiology of this condition remains to be understood, several theories have been developed in the past. Among them, the theory of dental lamina hyperactivity is still considered as the main reason for the development of mesiodens $[1,3,4]$. Several cases were shown to be associated with various craniofacial deformities, including cleft lip and palate, Down's syndrome, and cleidocranial dysostosis [3]. The mesiodens is commonly located at the midline of maxilla as a single, unerupted, and inverted entity. Few studies have shown that mesiodens can be found in the mandible, being bilaterally multiple, already erupting, and growing vertically [2-4]. It occurs more frequently in men than in women, with a ratio of $2: 1$ [1-5]. The prevalence of mesiodens in general population is $0.15-13.9 \%$ [3].

Mesiodens can be diagnosed by clinical and radiographic examinations. Clinical examination can reveal the presence of supernumerary tooth or teeth in the midline of maxilla, usually followed by a complication of the condition, including diastema $[2,3]$. Radiographic investigations are also performed to evaluate the posi-

\section{JOURNAL OF} STOMATOLOGY

AdDRESS FOR CORRESPONDENCE: Dr. Vera Julia, Department of Oral and Maxillofacial Surgery, Faculty of Dentistry, Universitas Indonesia, Salemba Raya No. 4, 10430, Jakarta, Indonesia, e-mail: vera.julia@ui.ac.id ReCEIVED: 14.05.2020 • ACCEPTED: 26.08.2020 • PUBlished: 30.10.2020 
tion of mesiodens and the condition of adjacent tooth. Periapical, panoramic, and occlusal radiographs can provide clear information regarding mesiodens [2-4]. In some cases, computed tomography (CT) and conebeam CT can also be used for diagnoses [2].

Various complications related to mesiodens include delayed eruption of permanent tooth, central diastema formation, and rotation of maxillary anterior teeth. Additionally, root resorption and cyst formation are the less commonly associated complications of mesiodens $[1,2]$. The main complaints of patient are based on these complications. The time of diagnosis is an important factor to be considered while managing a mesiodens. In some cases, a patient is diagnosed at the age of 6-10 years based on complaints. Furthermore, the appropriate time for the removal of a mesiodens remains controversial [3].

The characteristics of mesiodens must also be considered for the management of this condition $[1,3]$. Some types of mesiodens can be treated by a simple tooth extraction, whereas in some cases, the mesiodens must be removed using complicated methods, including surgical extraction. Surgical procedure can be followed by additional procedures for other complex complications, including orthodontic treatment $[1,4,5]$.

It is important to evaluate the optimal management of mesiodens based on the characteristics and complications of this condition. This study aims to review these factors and evaluate their association with the treatment methods used in previous studies.

\section{MATERIAL AND METHODS}

A literature search was performed by one author and included electronic database PubMed search for eligible studies published between January 2008 and December 2017. The question "How to determine the management of mesiodens considering the characteristics and complications of the condition?" guided the search strategy. Keywords, such as mesiodens, characteristics, complications, and management were applied to obtain an appropriate result. The final string used was mesiodens ${ }^{*}$ AND characteristics ${ }^{\star}$ AND complications ${ }^{\star}$ AND surgical manage- ment. Additional criteria, such as language and the time of publication, were included. The studies had to be published in English. PRISMA (Preferred Reporting Items for Systematic Reviews and Meta-Analysis) flowchart was also used. Those with review formats and topics that were unrelated to mesiodens were excluded (Table 1).

\section{RESULTS}

During the initial search, 330 articles were shown as results using the keyword "mesiodens", among which, 155 articles were published within the last 10 years. However, 60 out of 155 articles were excluded because of non-compliance with the aim of this study.

Figure 1 was formulated based on the PRISMA guidelines. After using the terms "mesiodens" AND "characteristics", we found 14 articles that were published within the last 10 years. Further detailed search using the keywords "mesiodens" AND “complication", and "mesiodens" AND "management" resulted in 18 and 28 articles, respectively, which were published within the last 10 years. Among a total of 60 articles, 45 articles relevant to our inclusion criteria were identified; however, only 30 full-text articles were available online for downloading (Table 2).

A majority of the studies (25 articles) were single or serial case reports, while the remaining 5 were retrospective studies (Tables 3 and 4). A review of case reports revealed 41 cases of mesiodens in 30 patients (Tables 4 and 5). They were determined based on the characteristics, chief complaints, complications, and management of the condition. Additionally, the characteristics and complications that needed to be considered for the management of mesiodens were determined in the five retrospective studies included in the present study (Table 3).

Most of the retrospective studies showed a prevalence rate of less than $1 \%$, except for the Patil study, which reported a prevalence of $>1 \%$ (Table 3 ). Although the majority of the studies reported a predilection for males, a female tendency was reported in the study by Colak et al. Likewise, the majority of these studies reported that the mesiodens occurs more frequently in young individuals (maximum age, 16 years). The most common characteri-

TABLE 1. Inclusion and exclusion criteria

\begin{tabular}{|c|c|c|}
\hline Criteria & Inclusion & Exclusion \\
\hline Period time & January 2008 to December 2017 & Any study outside the period \\
\hline Language & English & Non-English \\
\hline Type of article & Case report, original articles & Review \\
\hline Study focus & Mesiodens, complication, management & Unrelated to mesiodens, complication, and management \\
\hline Geographical area of interest & International studies & None \\
\hline Sample & Human, medical record & None \\
\hline Type of study & $\begin{array}{c}\text { Clinical report } \\
\text { Retrospective study }\end{array}$ & Review article \\
\hline
\end{tabular}




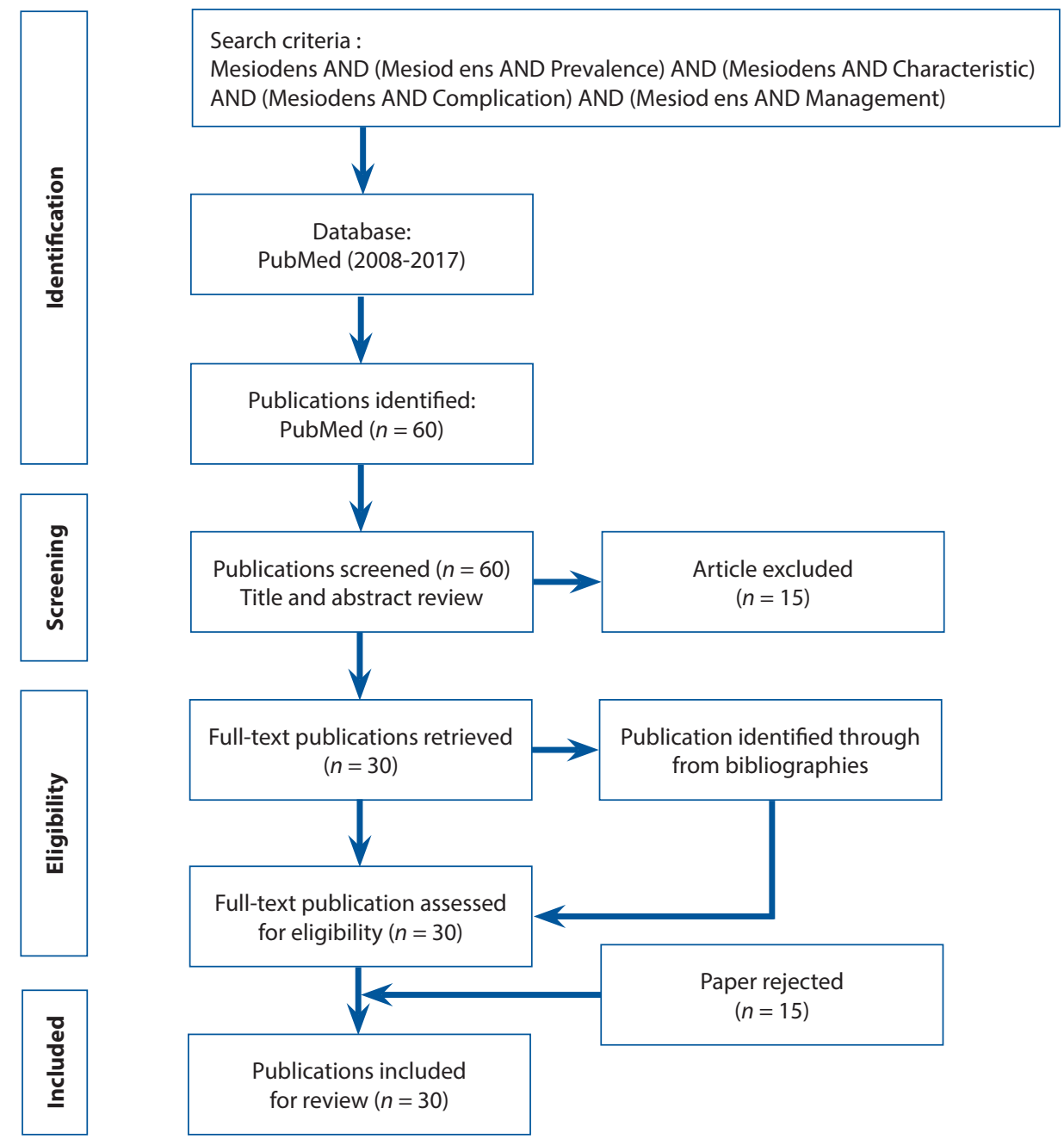

FIGURE 1. Flowchart of the study design

stics of mesiodens based on the studies were the number (single) and position (vertical) of the supernumerary tooth. However, differences in the eruption status and the shape of the crown were observed among the studies. Furthermore, only one study reported that most patients with mesiodens had no complications (Table 5).

\section{DISCUSSION}

As an abnormal additional tooth commonly located at the midline of maxilla, the mesiodens may occur as single or multiple entities. Variations in the eruption status, shape of the crown, and position have been observed. However, the etiology of this condition has not been clearly documented so far. Among the various theories proposed, dental lamina hyperactivity is considered as the most relevant [1-4].

All of the five retrospective studies reviewed in the current study indicated a prevalence of approximately $0.3-1.5 \%$, and a male to female ratio of $1.5: 2.1$ [1-5]. Only one out of these five retrospective studies stated that the mesiodens occurs more frequently in women than in men [4]. The mesiodens were usually found at the midline of maxilla. However, two case studies reported the anterior midline of mandible as the site of mesiodens, which are very rare occurrences $[6,7]$. The reason for this predilection for the maxilla remains unknown.

The mesiodens is usually found at a young age, especially in 6-9 years old individuals. However, few case reports have detected this anomaly in older patients (third to fifth decade) [8-11]. The chief complaints of young patients with mesiodens were delayed eruption, central diastema in the maxilla, and axial rotation of the permanent central incisors causing functional and esthetic issues. Older patients may not have been aware of the problem until accompanied by pain or swelling that may be related to the development of dentigerous cyst. Therefore, the timing of chief complaint may affect the timing of diagnosis of mesiodens.

The characteristics of mesiodens can be ascertained from the number, eruption status, shape of the crown, and the position in relation to the adjacent teeth. In a number of instances, the mesiodens occurs more fre- 


\begin{tabular}{|c|c|c|c|c|c|c|c|c|c|c|c|c|}
\hline 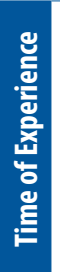 & 疍 & 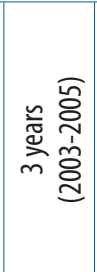 & 중 & 疍 & 츙 & 츙 & 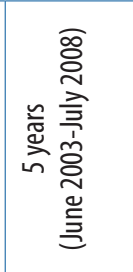 & 疍 & 츙 & 疍 & 층 & 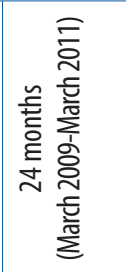 \\
\hline 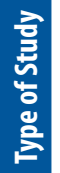 & 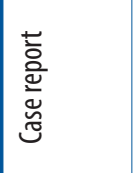 & 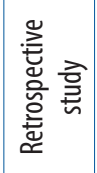 & 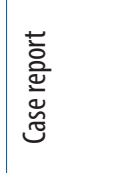 & 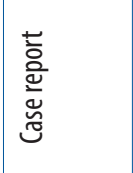 & 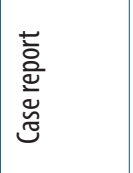 & 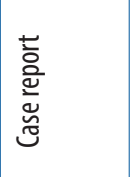 & 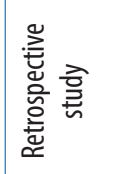 & 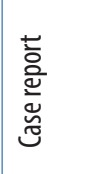 & 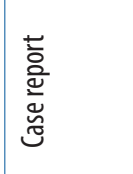 & 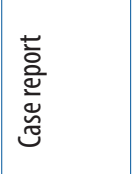 & 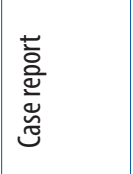 & 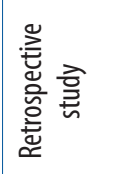 \\
\hline$=$ & - & 8 & - & - & - & - & $\circ$ & - & $m$ & $\sim$ & - & $\stackrel{\infty}{\llcorner}$ \\
\hline 䙷 & 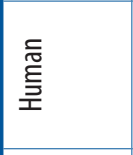 & 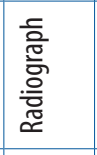 & $\begin{array}{l}\text { 点 } \\
\text { 咅 }\end{array}$ & \begin{tabular}{|l} 
㽕 \\
空
\end{tabular} & $\begin{array}{l}\text { 惡 } \\
\text { 产 }\end{array}$ & \begin{tabular}{|l} 
蔦 \\
空
\end{tabular} & 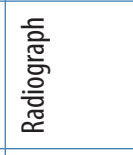 & \begin{tabular}{|l} 
疍 \\
产
\end{tabular} & \begin{tabular}{|l} 
点 \\
竞
\end{tabular} & \begin{tabular}{|l} 
竎 \\
产
\end{tabular} & \begin{tabular}{|l} 
惡 \\
塞
\end{tabular} & 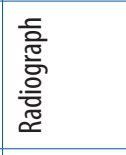 \\
\hline 寻 & 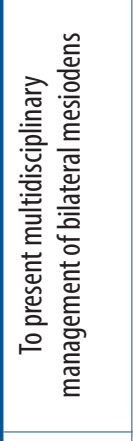 & 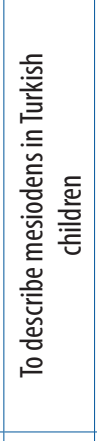 & 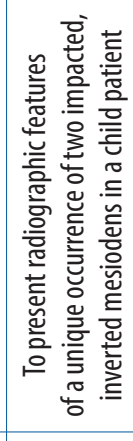 & 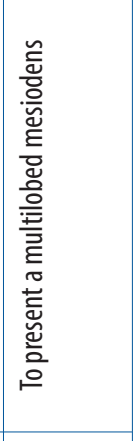 & 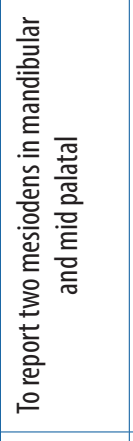 & 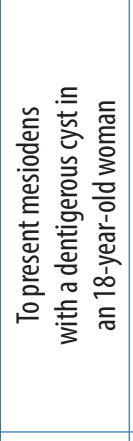 & 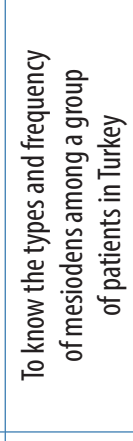 & 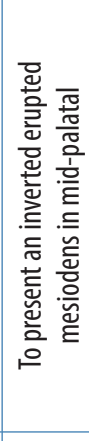 & 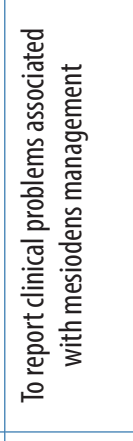 & 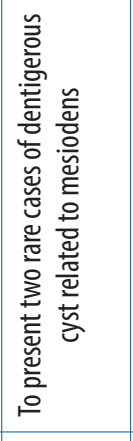 & 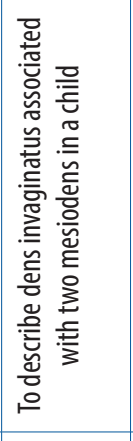 & 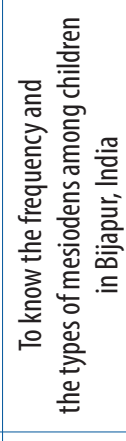 \\
\hline 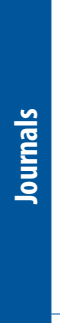 & 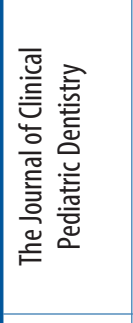 & 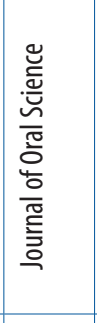 & 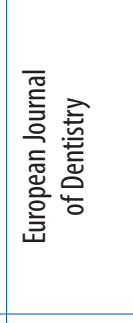 & 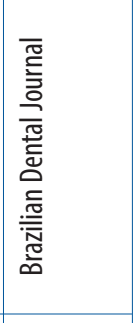 & 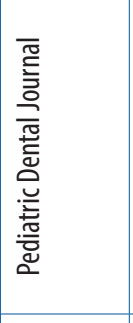 & 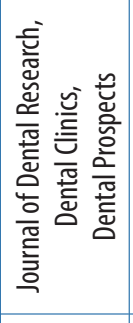 & 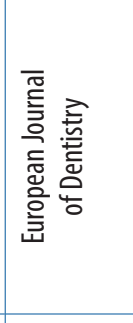 & 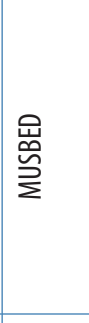 & 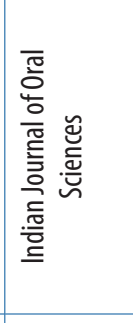 & 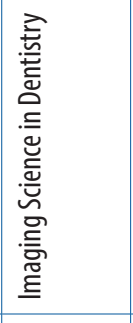 & 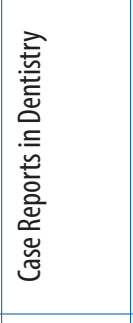 & 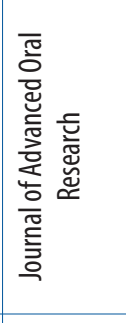 \\
\hline 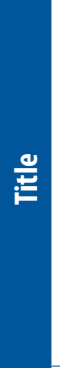 & 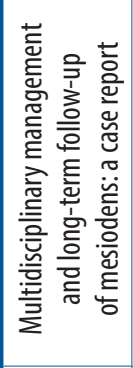 & 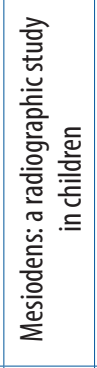 & 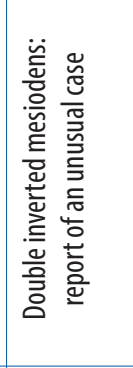 & 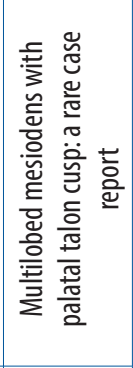 & 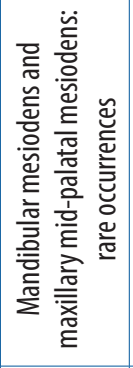 & 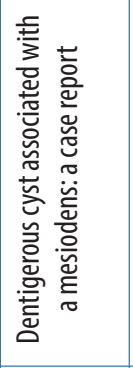 & 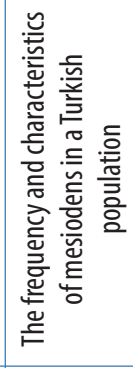 & 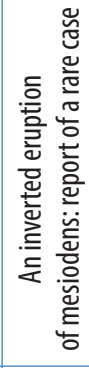 & 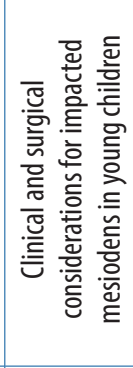 & 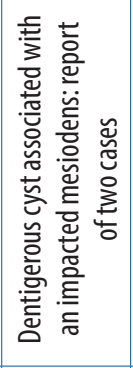 & 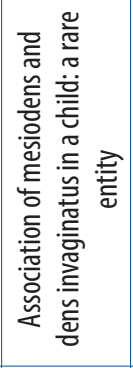 & 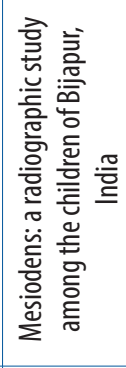 \\
\hline ‡ & 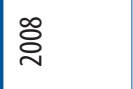 & 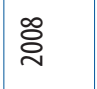 & 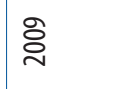 & 음 & ఇి & $\sim$ & 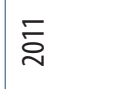 & $\tilde{\sim}$ & $\tilde{\check{\Sigma}}$ & సి & సి & 곡 \\
\hline$\frac{2}{0}$ & 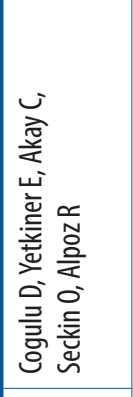 & 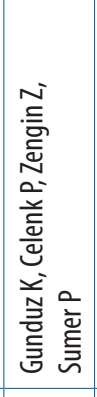 & 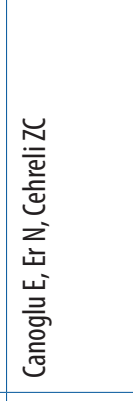 & 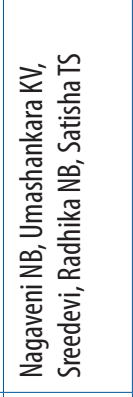 & 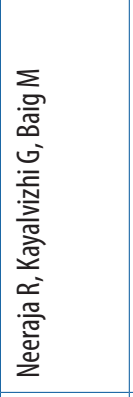 & 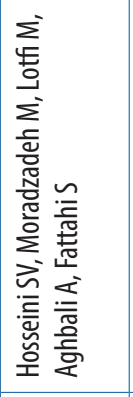 & 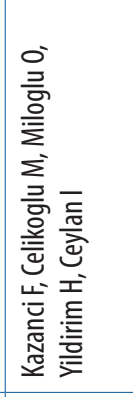 & 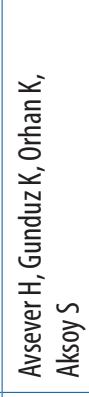 & 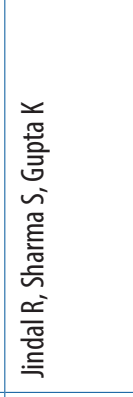 & 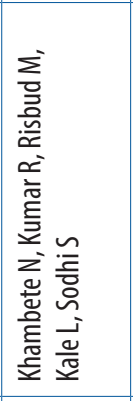 & 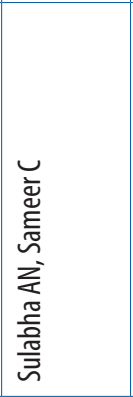 & 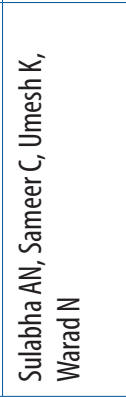 \\
\hline 을 & - & $\sim$ & $m$ & $\sigma$ & in & 6 & $r$ & $\infty$ & $a$ & 으 & $\approx$ & $\simeq$ \\
\hline
\end{tabular}




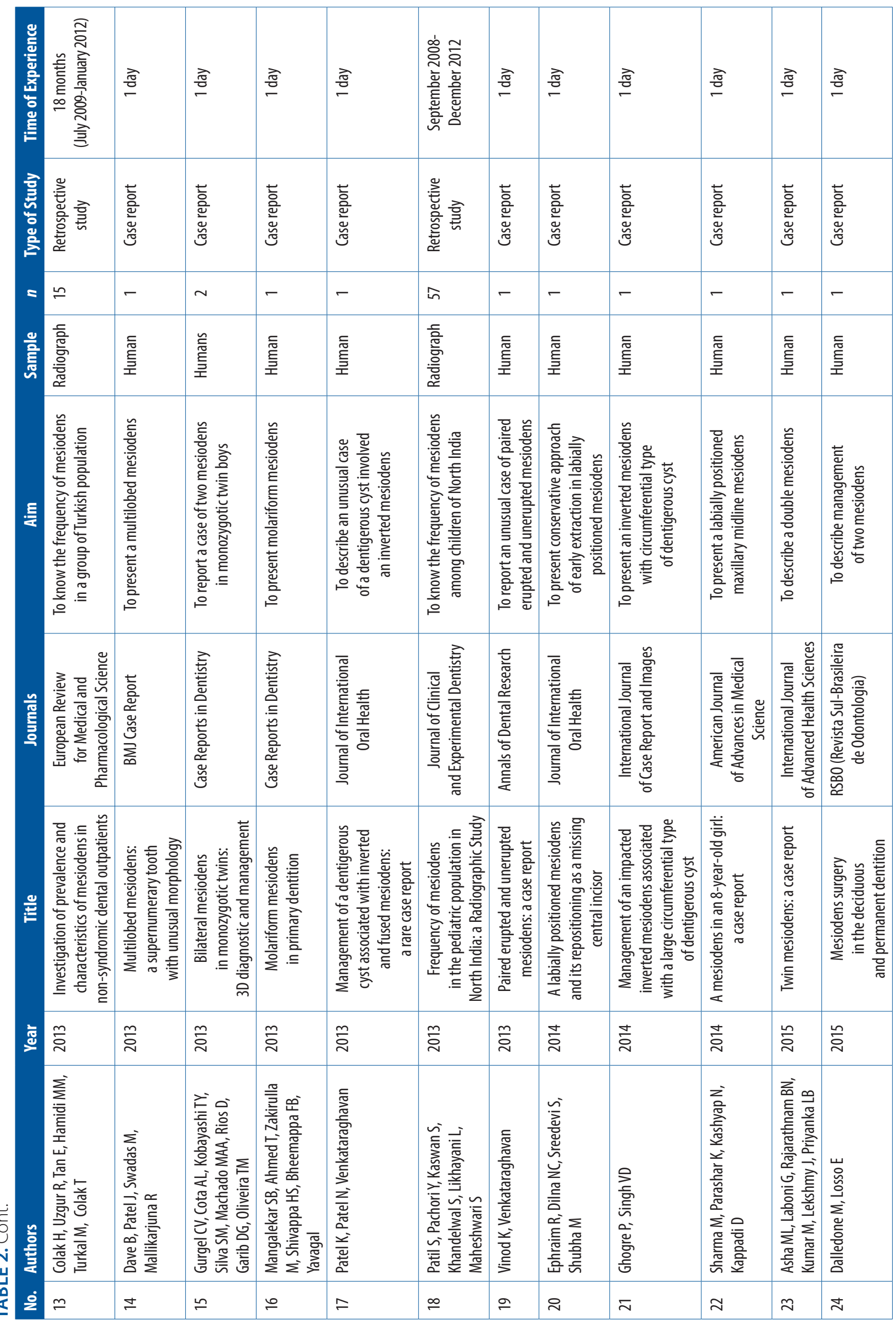




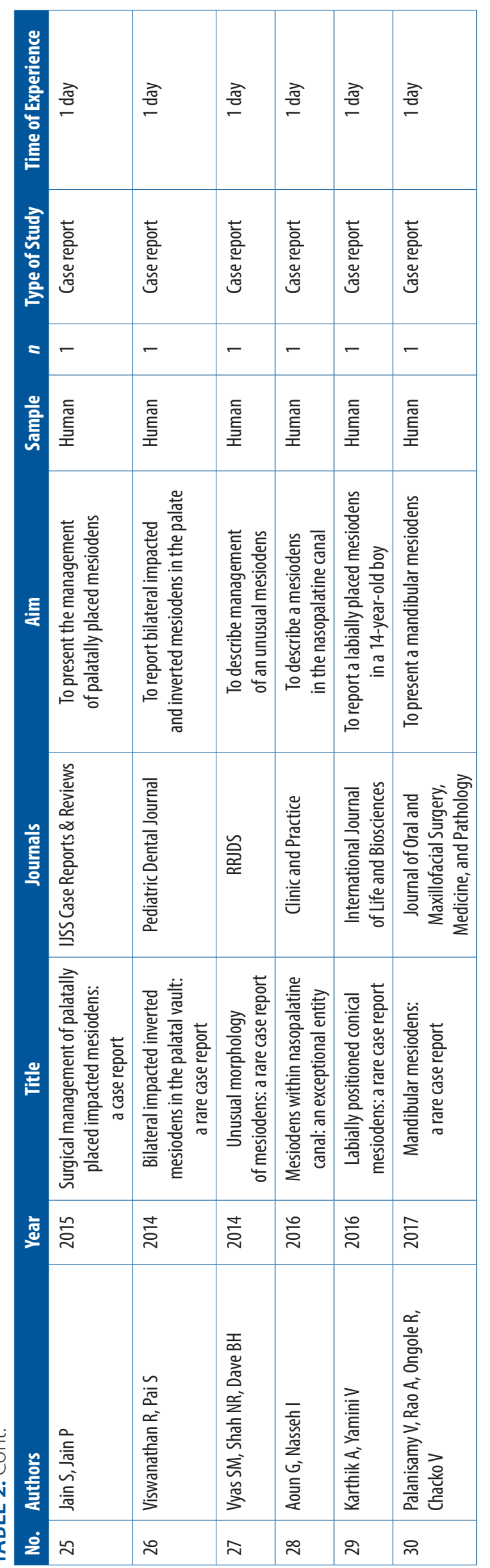

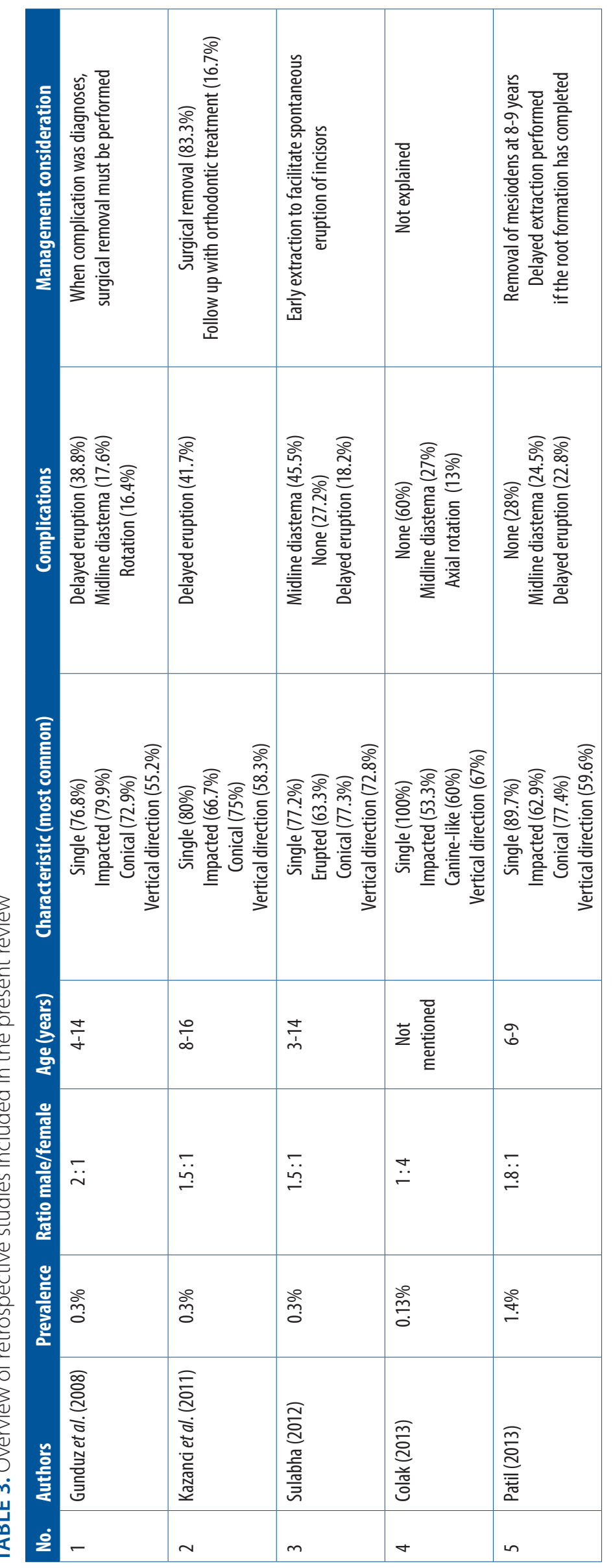




\begin{tabular}{|c|c|c|c|c|c|c|c|c|c|c|}
\hline 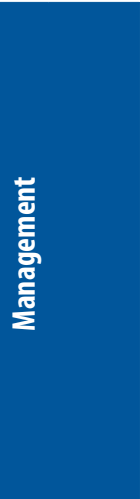 & 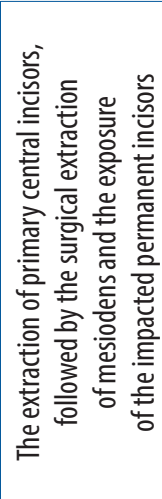 & 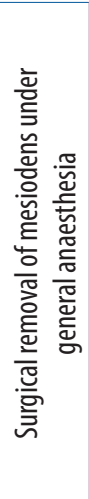 & 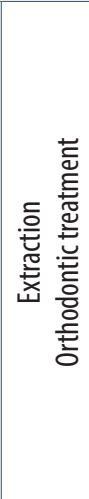 & 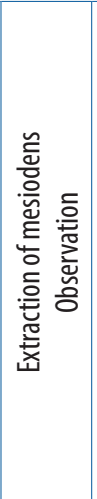 & 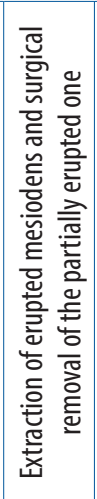 & 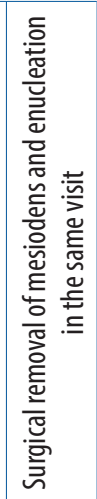 & 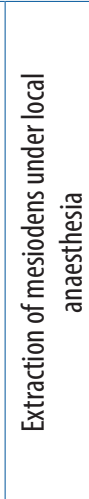 & 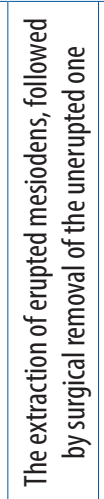 & 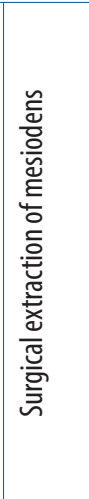 & 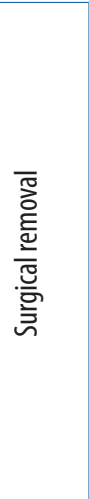 \\
\hline 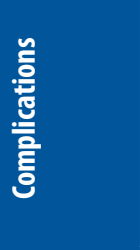 & 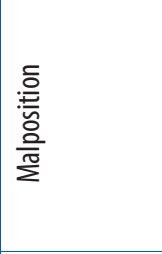 & 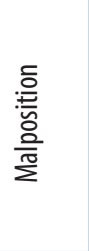 & 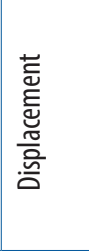 & 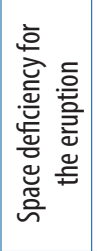 & 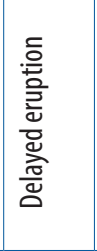 & 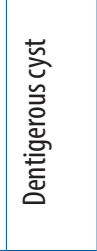 & 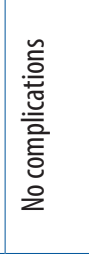 & 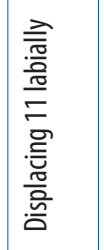 & $\begin{array}{l}\bar{E} \\
. \bar{E} \\
. \overline{0} \\
\frac{0}{0} \\
. \overline{0}\end{array}$ & 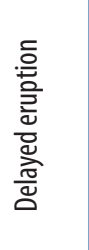 \\
\hline 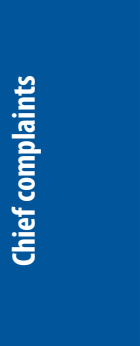 & 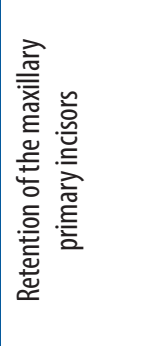 & 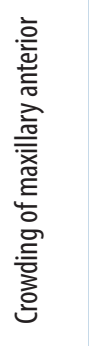 & 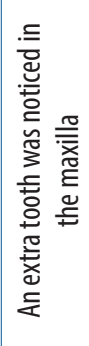 & 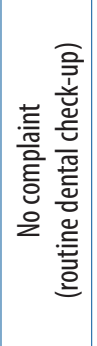 & 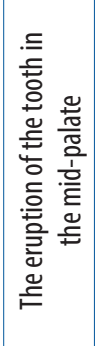 & 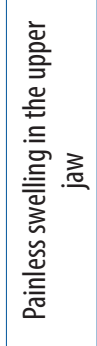 & 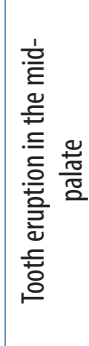 & 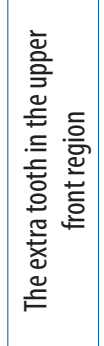 & 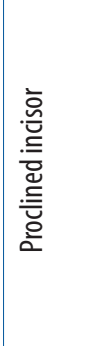 & 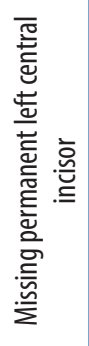 \\
\hline 辰 & 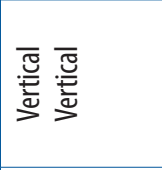 & 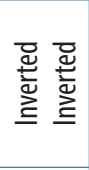 & 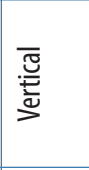 & 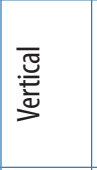 & 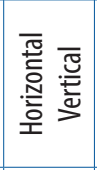 & 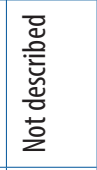 & 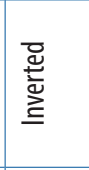 & 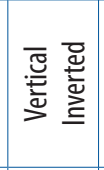 & 古 & $\sim \sim$ \\
\hline 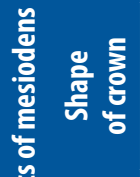 & 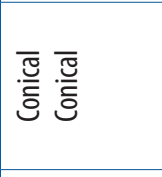 & 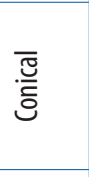 & 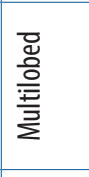 & 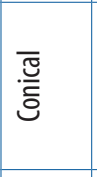 & 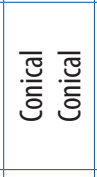 & $\begin{array}{l}\overline{\widetilde{\Xi}} \\
\text { ⿹्ర }\end{array}$ & 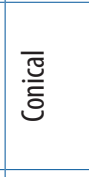 & 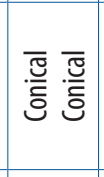 & 氖 & 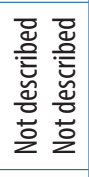 \\
\hline 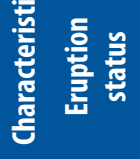 & $\begin{array}{l}\text { 总 } \\
\text { 总 } \\
\text { 兽 }\end{array}$ & 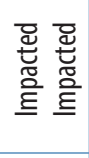 & 总 & 总 & 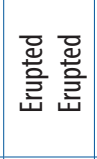 & 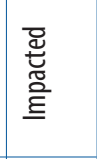 & $\begin{array}{l}\text { gू } \\
\text { 旁 }\end{array}$ & 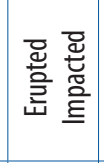 & 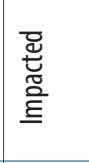 & 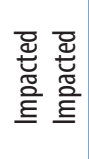 \\
\hline 兽 & $\frac{\circ}{\overline{\bar{a}}}$ & $\begin{array}{l}\frac{\circ}{\overline{0}} \\
\frac{\bar{a}}{\Delta}\end{array}$ & 离 & 离 & 言 & 莞 & 吾 & 高 & 竞 & $\frac{\stackrel{\circ}{\circ}}{\frac{\bar{z}}{\Delta}}$ \\
\hline 产 & $\infty$ & $\infty$ & $=$ & $n$ & $n$ & $\stackrel{\infty}{\stackrel{1}{2}}$ & $\stackrel{\infty}{\sim}$ & $a$ & $\circ$ & $a$ \\
\hline $\begin{array}{l}\frac{ \pm}{\mathrm{g}} \\
\mathrm{g}\end{array}$ & 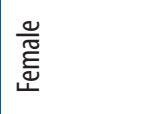 & $\frac{\Delta}{\sum^{\pi}}$ & $\frac{\Delta}{\tilde{N}}$ & 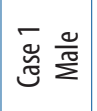 & 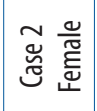 & 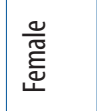 & $\frac{\frac{\Delta}{\pi}}{\sum}$ & $\bar{\Xi}$ & 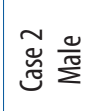 & 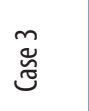 \\
\hline 产 & 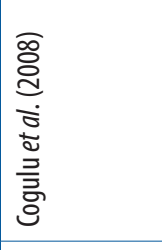 & 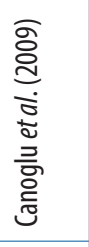 & 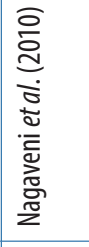 & 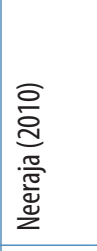 & & 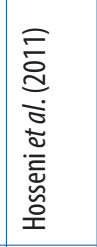 & 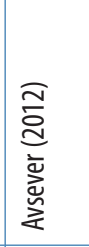 & 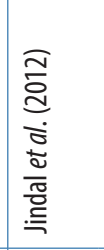 & & \\
\hline 운 & - & $\tau$ & $m$ & $\theta$ & & in & 0 & r & & \\
\hline
\end{tabular}


TABLE 5. Overview of mesiodens cases among the 25 case reports reviewed in this study

\begin{tabular}{|l|c|c|}
\hline Aspect & Criteria & $\boldsymbol{n}$ \\
\hline Age (total of 30 patients) & $\leq 16$ years & 23 \\
& $>16$ years & 7 \\
\hline Gender (total of 30 patients) & Male & 20 \\
& Female & 7 \\
& Not described & 3 \\
\hline Location (total of 41 mesiodens) & Maxilla & 39 \\
& Mandibula & 2 \\
\hline Number (total of 30 patients) & Single & 19 \\
& Double/Multiple & 11 \\
\hline Eruption status & Erupted & 15 \\
(total of 41 mesiodens) & Impacted & 26 \\
\hline Shape of crown & Conical & 26 \\
(total of 41 mesiodens) & Uncommon shaped & 5 \\
& Not described & 10 \\
\hline Position & Vertical & 19 \\
(total of 41 mesiodens) & Horizontal & 3 \\
& Inverted & 14 \\
& Not described & 5 \\
\hline
\end{tabular}

quently as a single entity (76-89\%) [1-5]. Most of the mesiodens were found to be fully impacted, followed by reports on partially and fully erupted mesiodens. Only one retrospective study reported that erupted mesiodens were more frequently seen $(63.3 \%)$ than other.

Several cases with rare characteristics were observed among the case reports that were discussed in the present study. Eleven out of 30 patients presented with double mesiodens and 3 of them had different eruption status (1 erupted tooth and 1 impacted tooth) $[6,12,19]$. Furthermore, 15 cases of erupted mesiodens were observed out of a total of 41 mesiodens [20-26].

Rare characteristics in terms of the shape of crown or the position or direction of the mesiodens were reported in some of the case reports. Mesiodens with multilobed or molariform crowns were also reported by several researchers. Likewise, horizontally placed and inverted mesiodens were also identified in several cases $[8,11,14,27]$. The shape of crown of the mesiodens may vary from conical, canine-, or incisor-like to tuberculated or round. The majority of articles reviewed in the present study reported the presence of conical-shaped (70\%) mesiodens. However, Colak et al. reported that the canine-like form was the most dominant (60\%) compared to other [4]. As for the position or direction of the mesiodens in relation to the adjacent tooth, it may be vertical, horizontal, or inverted. All studies reported the vertical position as the most common (55-72\%) [1-5].

Patients' primary complaint when they visited the dentist was that mesiodens were causing complications. However, Colak et al. and Patil et al. have reported the absence of complications in about $60 \%$ and $28 \%$ of patients with mesiodens, respectively $[4,5]$. In contrast, midline diastema and delayed eruption were the most common complications in some other retrospective studies [1-3]. Cyst formation does not appear to be a common complication in mesiodens, probably because the patients were examined at a young age (first and second decades) [1].

A number of cases with rarely seen complications, such as dentigerous cysts and root resorption, were reported in some of the case reports reviewed in this study. These types of complications generally occur in long-standing cases (during or after the second decade of life). If associated with a major complaint, the presence of a dentigerous cyst is usually characterized by the major complaints of pain and swelling.

The management of mesiodens can be viewed in terms of the timing (immediate or delayed) and technique (simple or surgical) of removal. Immediate treatment is usually considered for cases that are associated with complications, whereas delayed treatment usually occurs due to the age of patient, the absence of complaints, or when the roots of adjacent permanent teeth have not been fully formed. The age of $8-9$ years is considered as appropriate for the procedure using simple techniques or surgery with local anesthesia. In addition, the characteristics of mesiodens, such as the eruption status, must also be considered while performing simple procedures. An impacted mesiodens clearly requires surgery, although local anesthesia might be sufficient for the procedure. However, sometimes a vertically erupted mesiodens might require surgical extraction as long as there is no risk of damage to the adjacent fully erupted teeth [3].

Among the 25 case reports, 41 mesiodens in 30 patients were identified in this study. Immediate removal was carried out in 32 cases, whereas delayed removal was performed in the remaining 4 cases. This is understandable because almost all of these cases presented with complications or the risk of future complications, which could impact the complexity of treatment. The characteristics of mesiodens in relation to their management have not been well documented so far. However, in various cases when mesiodens has fully erupted, it can be removed by simple extraction, but an inverted mesiodens may require surgical removal to avoid damage to adjacent permanent teeth. The number of mesiodens (single or multiple) or form of the crown is generally not considered while determining the treatment method in these cases. On the other hand, the presence of complications, such as dentigerous cyst, can influence treatment planning.

Nevertheless, this review has to be interpreted with caution due to limitation of this study that has used only the PubMed as database information. Further review may use other databases.

\section{CONCLUSIONS}

In this study, we reviewed the management of 41 cases of mesiodens based on their characteristics and associat- 
ed complications. The chief complaints of patients were dependent on the complications associated with mesiodens. The mesiodens was generally treated when the patient presented to the dentist with a chief complaint. Furthermore, the characteristics of mesiodens (especially, the eruption status and position) were likely to be considered during the management of this condition. Unfortunately, it was not possible to determine the significance of the characteristics and complications on the timing and surgical management of the mesiodens from the 30 articles that were reviewed in this study.

\section{ACKNOWLEDGMENT}

The authors thank Dr. Diah Ayu Maharani (Department of Preventive and Public Health Dentistry, Faculty of Dentistry, Universitas Indonesia) for her invaluable comments for this study.

\section{CONFLICT OF INTEREST}

The authors declare no potential conflicts of interest with respect to the research, authorship, and/or publication of this article.

\section{References}

1. Gunduz K, Celenk P, Zengin Z, Sumer P. Mesiodens: a radiographic study in children. J Oral Sci 2008; 50: 287-291.

2. Kazanci F, Celikoglu M, Miloglu O, Yildirim H, Ceylan I. The frequency and characteristics of mesiodens in a Turkish patient population. Eur J Dent 2011; 5: 361-365.

3. Sulabha AN, Sumeer C, Umesh K, Warad NM. Mesiodens: a radiographic study among the children of Bijapur, India. J Adv Oral Res 2012; 3: 15-20.

4. Colak H, Uzgur R, Tan E, Hamdi MM, Turkal M, Colak T. Investigation of prevalence and characteristics of mesiodens in a non-syndromic 11256 dental outpatients. Eur Rev Med Pharmacol Sci 2013; 17: 2684-2689.

5. Patil S, Pachori Y, Kaswan S, Khandelwal S, Likhyani L, Maheshwari S. Frequency of mesiodens in the pediatric population in North India: a radiographic study. J Clin Exp Dent 2013; 5: 223-226.

6. Neeraja R, Kayalvizhi G, Baig M. Mandibular mesiodens and maxillary mid-palatal mesiodens: rare occurence. Pediatr Dent J 2010; 20: $186-188$

7. Palanisamy V, Rao A, Ongole R, Chacko V. Mandibular mesiodens: a rare case report. J Oral Maxillofac Surg Med Pathol 2016; 29: 163-165

8. Khambete N, Kumar R, Risbud M, Kale L, Sodhi S. Dentigerous cyst associated with an impacted mesiodens: report of 2 cases. Imaging Sci Dent 2012; 42: 255-260.

9. Patel K, Patel N, Venkatarraghavan K. Management of a dentigerous cyst associated with inverted and fused mesiodens: a rare case report. J Int Oral Health 2013; 5: 73-77.

10. Ghogre P, Singh VD. Management of an impacted inverted mesiodens associated with a large circumferential type of dentigerous cyst: a rare case report with one-year follow-up. Int J Case Rep Images 2014; 5: 80-85.

11. Aoun G, Nasseh I. Mesiodens within the nasopalatine canal: an exceptional entity. Clin Pract 2016; 6: 119-121.
12. Cogulu D, Yetkiner E, Akay C, Seckin O, Alpoz R. Multidisciplinary management and long-term follow-up of mesiodens: a case report. J Clin Pediatr Dent 2008; 33: 63-66.

13. Canoglu E, Er N, Cehrel ZC. Double inverted mesiodentes: report of an unusual case. Eur J Dent 2009; 3: 219-223.

14. Jindal R, Sharma S, Gupta K. Clinical and surgical considerations for impacted mesiodens in young children: an update. Indian J Oral Sci 2012; 3: 94-98.

15. Sulabha AN, Sameer C. Association of mesiodentes and dens invaginatus in a child: a rare entity. Case Rep Dent 2012; 2012: 198032.

16. Gurgel CV, Cota ALS, Kobayashi TY, et al. Bilateral mesiodens in monozygotic twins: 3D diagnostic and management. Case Rep Dent 2013; 2013: 193614.

17. Vinod K, Venkataraghavan K. Paired erupted and unerupted mesiodens: a case report. Ann Dent Res 2013; 3: 15-20.

18. Asha ML, Laboni G, Rajarathnam BN, et al. Twin mesiodens: a case report. Int J Adv Health Sci 2015; 2: 18-21.

19. Viswanathan R, Pai S. Bilateral impacted inverted mesiodentes in the palatal vault: a rare case report. Pediatr Dent J 2014; 25: 26-28.

20. Nagaveni NB, Umashankara KV, Reddy BP, Radhika NB, Satisha TS. Multi-lobed mesiodens with a palatal talon cusp: a rare case report. Braz Dent J 2010; 21: 375-378.

21. Hosseni SV, Moradzadeh M, Lotfi M, Aghbali AA, Fattahi S. Dentigerous cyst associated with a mesiodens: a case report. J Dent Res Dent Clin Dent Prospects 2011; 5: 76-78.

22. Avsever H, Gunduz K, Othan K, Aksoy S. An inverted eruption of mesiodens: report of a rare case. Musbed 2012; 2: 37-39.

23. Dave B, Patel J, Swadas M, Mallikarjuna R. Multilobed mesiodens: a supernumerary tooth with unusual morphology. BMJ Case Rep 2013; 2013: bcr2012008088.

24. Mangalekar SB, Ahmed T, Zakirulla M, Shivappa HS, Bheemappa FB, Yavagal C. Molariform mesiodens in primary dentition. Case Rep Dent 2013; 1-5.

25. Vyas SM, Shah NR, Dave BH. Unusual morphology of a mesiodens: a rare case report. Res Rev J Dental Sci 2014; 2: 39-42.

26. Karthik A, Yamini V. Labially positioned conical mesiodens: a rare case report. Int J Life Biosci 2016; 2: 107-109.

27. Ephraim R, Dilma NC, Sreedevi S, Subha M. A labially positioned mesiodens and its repositioning as a missing central incisor. J Int Oral Health 2014; 6: 114-117.

28. Sharma M, Parashar K, Kasyap N, Kappadi D. A mesiodens in an 8 year old girl-a case report. Am J Adv Med Sci 2014; 2: 47-52.

29. Dalledone M. Mesiodens surgery at deciduous and permanent dentition. RSBO 2015; 12: 94-97.

30. Jain S, Jain P. Surgical management of palatally placed impacted mesiodens: a case report. IJSS Case Rep Rev 2015; 1: 70-72. 